\title{
A Stochastic activity-based approach for forecasting occupant-related energy consumption in residential buildings
}

Toufic Zaraket, Bernard Yannou, Yann Leroy

\author{
Ecole Centrale Paris \\ Laboratoire Genie Industriel \\ Chatenay-Malabry, France \\ toufic.zaraket@ecp.fr, bernard.yannou@ecp.fr, \\ yann.leroy@ecp.fr
}

\author{
Stephanie Minel, Emilie Chapotot \\ ESTIA \\ Technopole Izarbel \\ Bidart, France \\ s.minel@estia.fr, e.chapotot@estia.fr,
}

\begin{abstract}
Building occupants are considered as a major source of uncertainty in energy modeling nowadays. Yet, industrial energy simulation tools often account for occupant behavior through some predefined scenarios and fixed consumption profiles which yield to unrealistic and inaccurate predictions. In this paper, a stochastic activity-based approach for forecasting occupant-related energy consumption in residential buildings is proposed. First, the model is exposed together with its different variables. Second, a direct application of the model on the domestic activity "washing laundry" is performed. A number of simulations are performed and their results are presented and discussed. Finally, the model is validated by confronting simulation results to real measured data.
\end{abstract}

Key words: Energy consumption, residential building, energy model, household profile, activity, occupant behavior, consumption variability.

\section{INTRODUCTION}

The building sector is a substantial energy consumer and pollution source in most countries. It is responsible for important shares, ranging between 16 and 50 percent, of national energy consumptions worldwide [1,2]. In France, buildings account for around $43 \%$ of the total national energy consumption and $25 \%$ of total $\mathrm{CO} 2$ emissions [3]. Reducing these consumptions and emissions is therefore a vital step towards sustainable development.

Similarly to other developed countries, French authorities have established recently a number of standards and regulations so to promote sustainable development in the building sector. An example of such regulations is the RT 2012, standing for "Réglementations Thermiques 2012" (i.e. Thermal Regulation). This regulation is an ambitious step towards promoting green buildings since it plans to divide by three the energy consumption of new buildings starting from the end of year 2012. As a result of such norms, building constructors are tending more and more to construct energy-efficient and green buildings. Moreover, a so-called "performance contract", which is a performance commitment between building constructors and owners, is a new market expectation emerging in France. By this contract, constructors commit to deliver an eco-efficient building and to guarantee its performance for a number of years after handover. This shift towards constructing low-consuming and nearly zero energy buildings, lead to further requirements with regard to performance and sustainability and thus caused the design process of buildings to be more complex. Therefore, a better comprehension and integration of building performance determinants into the design of buildings, especially in the very early phases, has become essential.

In general, the energy performance of a building is governed by various parameters, such as its physical characteristics, its internal services systems and equipments, its external environment and most importantly its occupants [4,5]. While energy simulation tools can assess, with a good precision, the influence of other parameters, yet they are still facing limitations in modeling occupants' energy consumption behaviors [6]. In fact, energy simulation tools, such as EnergyPlus, eQUEST, ESP-r and TRNSYS, focus primarily on the structural behavior of buildings and their relations to specific environmental conditions while taking into account insufficiently the role of the occupants [7]. This simplification of occupants' influence is eventually leading to unrealistic assumptions about average user preferences and behaviors [8]. For these reasons, energy and buildings experts are recently devoting considerable efforts for finding tools, techniques and approaches that enable them to better understand, interpret and model occupants influence on whole building performance. 


\subsection{Occupants and residential energy consumption}

The residential sector consumes secondary energy, which is used by occupants in suitable form for their domestic activities. Several studies pointed out the major end-use groups of secondary energy such as space heating, space cooling, domestic hot water, as well as appliances and lighting [9]. Energy use of buildings is strongly dependant on systems operation and general behavior of occupants. According to Page et al. [10] and Robinson [11], the influence of occupants can be translated by their presence, the actions they perform (activities such as cooking, using light, etc.), as well as their interactions with the controls of inherent building systems designed for adjusting indoor environment. According to Robinson [11], the most complex processes taking place within buildings are those that result from human behavior. Lutzenhiser et al. [12] confirm that household attributes such as income, education, family size, occupation hours, and household are highly influential on energy consumption. Guerin et al. [13] identify household income, age, education of owners, home ownership, desire for comfort, and energy conservation incentives as influencing factors. McLoughlin et al. [14] identify the number of occupants, disposable income, head-of-household age, tenure type, social group, education level, and appliance ownership as most influencing factors on residential energy consumption.

Energy consumption can vary dramatically between different households. This variation is due to the variability in occupant profiles (socio-demographic and economic attributes) which leads to variability in equipment possession and energy consumption patterns. According to Swan and Ugursal [15], occupant behavior in residential buildings varies widely and can impact energy consumption by as much as $100 \%$ for a given dwelling. Pachauri [16] concludes that the total household income level is the most important explanatory variable causing variation in energy requirements across Indian households.

For these reasons, building and energy experts manifest their need for more precise methods for modeling occupants influence on whole building performance. Such models should result in better energy estimation results and therefore in better building designs and marketing offers.

\subsection{Modeling energy consumption in residential buildings}

A number of techniques and approaches have been developed to address the issue of modeling energy consumption in residential buildings. According to Swan and Ugursal [15], the two major streams of approaches identified are top-down (econometric or technological) and bottom-up (statistical or engineering) approaches, with each of them comprising a number of scientific techniques. For more knowledge about these approaches, the reader is referred to Swan and Ugursal [15] and McLoughlin et al. [14].

In general, the research on occupant-related residential energy consumption can be divided into two groups of methods. The first group consists of using real sub-metering data in order to derive representational load or diversity profiles of occupants energy use, and thus deduce estimates of buildings' energy consumption. The second group of studies focuses on the development of approaches that can better represent occupants' behavior. Such models aim at simulating occupancy patterns and various energy-load schedules by using stochastic approaches [17]. Although such models can generate representative load profiles and provide some insights about occupants' role in energy consumption, yet they do not depict the complex phenomena of occupant behavior. Instead of using sub- metering data, the studies from the second group use other source of information, namely the time use surveys (TUS). The latter can be defined as large-scale time-use surveys conducted at the national level. Each TUS record contains information on 24-hour period of activities of a given individual [18]. A number of authors have used such surveys so that to depict and model occupants' daily energy use. By using stochastic techniques such as Monte Carlo Markov chains (MCMC), daily activity patterns of energy consumption can be derived from TUS data.

Tanimoto [19] proposed a stochastic approach for residential coolingload calculations. The same author develops later a method to simulate the load schedules for appliances, lighting, and hot water [20]. Tanimoto does not offer any discussion regarding the strength and limitation of his approach. Richardson et al. [21] introduce a Markovchain technique to generate synthetic active occupancy patterns, based upon time-use surveys in the United Kingdom. The stochastic model proposed by Richardson et al. provides a mapping between occupant activity (state) and appliance use, creating thus highly resolved synthetic energy demand data. In their results, Richardson et al. [21] find good match between occupancy profiles yielded by the model and real profiles taken from the TUS data. Based on their occupancy model, the same authors also develop a domestic electricity demand model [22]. Widén and Wäckelgård [23] develop a high-resolution stochastic model of domestic activity patterns and electricity demand in Sweden. They identify nine different electricity-dependent activities such as sleeping, cooking, dishwashing, cloth washing, TV and others. The authors associate then each of these activities to its corresponding domestic appliance(s). By defining load patterns for each appliance, Widén and Wäckelgård estimate the total electricity demand per household. The authors show that realistic demand patterns can be generated from these activity sequences. Muratori [24] use heterogeneous Markov chains to model domestic activity patterns of individuals, and to predict energy consumption of households. Subbiah [25] uses American TUS data for developing a disaggregated energy demand-modeling framework that estimates energy demand profiles based on individual-level and building-level energy-consuming activities. Subbiah [25] claims that his model can result in better results than other TUS-based models since it can account for interactions between household members and that it computes domestic activities at both individual and household levels.

Recently, other approaches stemming from artificial intelligence domain have started to be applied for modeling the dynamic aspects of energy consumption in buildings. Kashif et al. [8] proposed a conceptual framework to simulate dynamic group behavior by using an agent-based approach. The authors used this framework to predict the energy consumption of a household by simulating the interactions between inhabitants living in the same home. Quijano et al. [26] proposed an agent-based simulation platform called SMACH (multiagent simulation of human behavior) for assessing the impact of the adaptive behavior of various electrical appliances on the overall consumption of dwellings. The human agents imitating individuals' behaviors are modeled from observations in the real world of some volunteer families. As concluded by Quijano et al., the major limitation of their work is that the different strategies have not been tested in a real environment and that it would be difficult to identify the activity of each individual at every moment [26].

\subsection{Research gaps in occupant-related energy consumption models}

Given our research perspectives, a number of shortcomings associated to models found in literature review are identified. Firstly, 
even though most of the models highlight a relatively high number of energy consumption determinants related to occupants (such as the income, age, etc.), yet they are still too far simplistic with representing these determinants. In most of these models, the main variable considered for representing households' attributes is the number of occupants. This means that such models cannot assess variability of energy consumption for instance between two households having the same number of occupants but of different socio-economical attributes. Secondly, there has been little published work for generating energy demand profiles with a very fine granularity. The models in literature do not provide the complete ability to quantify energy consumption at the level of a specific household or a specific individual according to their social, demographic, and economical characteristics. Thirdly, most of the published models are based either on monitored consumption data or on time use surveys. The reliability of these sources of data can be criticized since it represents a part of the population, and not the whole population. For instance, time use surveys only consider activity schedules of the individuals who responded to the survey; thus, other household members are considered as having same activity schedules which is not rational and can lead to unrealistic energy demand predictions. Fourthly, published models do not present a clear view on how domestic activities can be carried out by and shared among household members. The aggregation of individual activity quantities at the level of the household has not clearly tackled. For instance, if two or more individuals are watching $\mathrm{TV}$ at the same time, the energy consumption of the appliance must be counted only once.

\section{A STOCHASTIC ACTIVITY-BASED ENERGY CONSUMPTION MODEL PROPOSAL}

The present paper does not intend to model aggregated or typical behavior of building occupants, neither to develop dynamic models that calculate energy consumption on the basis of daily time-steps. However, it proposes a parametric predictive model which takes a certain household profile with certain attributes as input and gives its corresponding energy consumption spectrum as output. The main advantages of such a model are its capability to reveal the variability in consumption values among different households, and to provide accurate energy demand spectrums as a function of households' attributes.

A stochastic bottom-up model using an activity-based approach is thus adopted. Such an approach requires knowledge about occupants and their energy use patterns. Thus information regarding households' characteristics and their lifestyles are needed. Activity-based approach means that energy consumption of a household is estimated by summing up the energy use of different activities performed (such as cooking, washing clothes, etc.). The stochastic nature of the model is due to the probabilistic mapping established between household attributes from one side (household type, number of occupants, etc.) and the corresponding appliance ownership, appliance characteristics and power rating, and activity quantities from the other side. In order to establish these stochastic relations, a fairly sufficient number of households' characterizing attributes is taken into account.

The structure of the proposed SABEC (Stochastic Activity-Based Energy Consumption) model is represented in Figure 1. The different objects of the model are explained in the following section. This model lies on two major hypotheses which are discussed further in this paper. First, for deriving an activity quantity per household from an estimation of the activity quantities per individuals, cumulative summation may be assumed for a given activity but of course the sharing of activity or economies of scale may diminish this basic summation. Second, activities in a dwelling must be enounced in such a way that they do not overlap on each other and the cumulative sum of energy consumed per each activity may be used to globally assess energy consumption of a household in a dwelling.

\subsection{Households' and individuals' attributes}

A household comprises one or more individuals living in the same dwelling and is characterized by a number of attributes. Some characteristics of a household are represented by those of its reference person (RP). The definition of reference person, also called household head, is widely adopted in scientific literature [14,27,28] and national statistics [29]. The reference person is defined as the elder economically-active individual among household adults, and thus taken as representative of households' socio-economic status. Therefore, the same definition of reference person is adopted in this paper. Moreover, the household type can be single, one-parent family, couples without children, or couples with children. The attributes describing individuals and households are chosen based on literature review and statistical studies. In addition to these variables, we introduce an important intermediary variable called the environmental awareness. The latter represents individuals' attitudes towards purchasing energy efficient appliance as well as their energy consumption patterns. Literature review and statistical studies show that the environmental awareness of a household is directly related to three main attributes which are the RP's age and education level, and household's total income [28,30]. The list of occupant-related attributes is illustrated in Table 1, where their detailed distributions over the French population are taken from national statistics [30,31].

Table 1. INDIVIDUALS AND HOUSEHOLDS ATTRIBUTES

\begin{tabular}{ll}
\hline Individual attributes & Household attributes \\
\hline Age & Household type \\
Gender & Number of adults $(>18$ years $)$ \\
Activity status & Number of children $(<18$ years $)$ \\
Socio-professional class & Household's total income \\
Education level & RP's age \\
Income & RP's activity status \\
& RP's socio-professional class \\
& RP's education level \\
\hline
\end{tabular}

Therefore, given the initial characteristics of household members, household's representative attributes can be determined. The environmental awareness of the household is determined by using the three determinant variables: household's total income $\left(I_{H H}\right)$, reference person's age $\left(A G_{R P}\right)$ and education level $\left(E L_{R P}\right)$. The probability for a given household to have a high level of environmental awareness (HEA) given each of the preceding variables separately is drawn from a French statistical study conducted by Maresca et al.[30]. Environmental awareness level is evaluated on a scale of 1 to 5. High environmental awareness corresponds thus to 4 and 5 levels, while Low environmental awareness is between 1 and 3 [30].Combining these three probabilities enables us to compute the probability for a household to have a high environmental awareness $P\left(H E A_{H H}\right)$ as shown in equation 1 . The formula for calculating the joint conditional probability of an event given three or more dependent events is adopted from Journel [32].

$P\left(H E A_{H H}\right)=P\left(H E A \mid A G_{R P}, I_{H H}, E L_{R P}\right)$

\subsection{Appliance ownership rate}

The appliance ownership rate for a given household is estimated as a function of three main variables: Household type $H_{\text {Type }}$, Reference person's age $\left(A G_{R P}\right)$, and reference person's socio-professional 
category $S P C_{R P}$. The conditional probability of having an appliance, given each of the three variables separately, is taken from national French statistics [33] Then by using same joint probability formula as earlier, the probability for a household to have certain appliance $P(A P)$ can be estimated as shown in equation 2 .

$$
P(A P)=P\left(A P \mid H H_{\text {type }}, S P C_{H H}, A G_{R P}\right)
$$

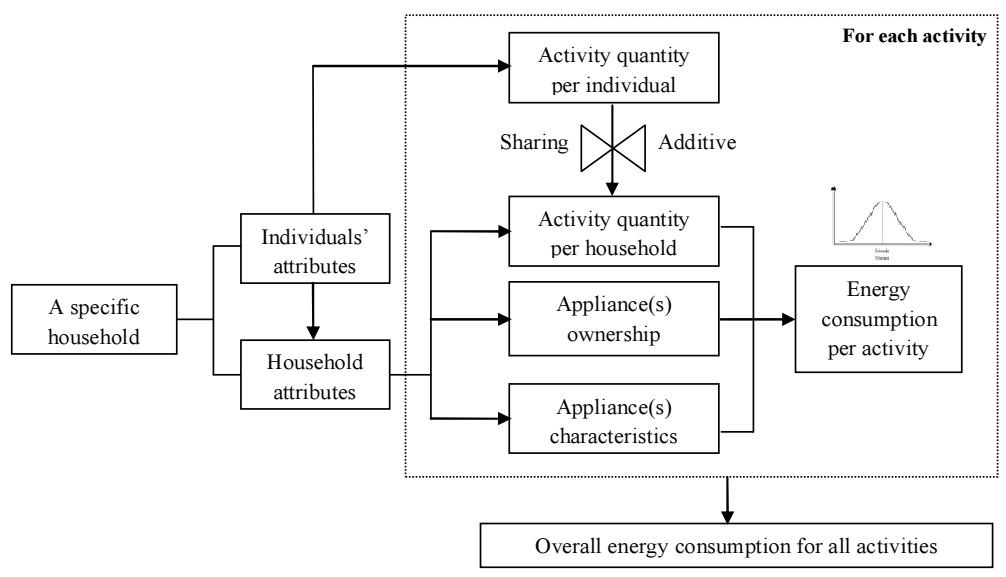

Figure 1: SABEC model structure

\subsection{Appliance characteristics}

The characteristics of an electrical appliance are mainly its technology (e.g. for televisions: LCD, CRT, and plasma) and energy rating. A domestic appliance is said to be energy-efficient if it consumes less-energy than other devices providing the same function or service. The energy efficiency of an appliance is rated in terms of a set of energy classes (labels) ranging from $\mathrm{A}$ (recently $\mathrm{A}++$ ) which is the most efficient to $G$ which is the least efficient.

The ownership probability of an energy-efficient appliance is considered as a function of three main variables: reference person's age $\left(\mathrm{AG}_{\mathrm{RP}}\right)$, household's environmental awareness level $\left(\mathrm{EAL}_{\mathrm{HH}}\right)$, and household's total income $\left(\mathrm{I}_{\mathrm{HH}}\right)$. The conditional probability of having an energy-efficient appliance, given each of the three variables separately, is taken from an important French study conducted by CREDOC $^{1}$ [30]. For example, Table 2 shows this probability as a function of households' monthly income.

Thus, the joint probability for a household to possess an energyefficient appliance $P(E A P)$ is given as shown in equation 3 .

$P(E A P)=P\left(E A P \mid A G_{R P}, I_{H H}, E A L_{H H}\right)$

Table 2. CONDITIONAL PROBABILITY OF HAVING ENERGYEFFICIENT APPLIANCES GIVEN THE INCOME [30]

\begin{tabular}{cc}
\hline $\begin{array}{c}\text { Household's total income } \\
\text { (Euros/month) }\end{array}$ & $\begin{array}{c}\text { Probability of owning an } \\
\text { energy-efficient appliance }\end{array}$ \\
\hline $700-1000$ & 0.31 \\
$1000-1500$ & 0.50 \\
$1500-2000$ & 0.62 \\
$2000-3000$ & 0.70 \\
$3000-4500$ & 0.80 \\
4500 or more & 0.70 \\
\hline
\end{tabular}

\subsection{Estimating activity quantities per household}

In order to determine the quantity of a given activity for a given household, a quantification unit namely the "activity's service unit" is

${ }^{1}$ CREDOC : French research centre for the study and monitoring of living standards defined. This definition is based on that of the functional unit in life cycle analysis (ISO 14044). For example, the service unit of the activity "watching TV" is defined to be the duration of watching TV in minutes per day. Such data can be obtained based on national statistics and studies. Using this service unit together with the power rating of the equipment used, the energy consumption for activity can thus be estimated.

The service unit of an activity at the household level is derived from individual service units. For this reason, two types of activities are distinguished: additive activities whose aggregated service unit is simply the sum of service units per individual (e.g. bathing), and shared activities whose service unit is not additive, but rather shared by two or more family members (e.g. watching TV). This sharing part can be accounted for either by using statistical data about sharing coefficients, if data is available, or by defining heuristic logics, expressing the degree to which people of a household share an activity. This yields to the estimation of the total service unit of the household for a given activity $\left(\mathrm{ASU}_{\mathrm{HH}}\right)$. The aggregation function of the service unit differs as a function of the activity.

\subsection{Estimating energy consumption of an activity}

The energy consumption of an activity for a given household is estimated based on the variables presented above. Given the probabilistic nature of model variables, Monte-Carlo technique is used for running simulations. At each run, random variables are generated, based on probabilistic distributions, to estimate: (1) the environmental awareness level of the household (EAL), (2) the ownership rate of appliances (AP) (3) the energy-efficiency of appliances (EAP), and (4) the appliance technology.

Individual service units of a given activity are obtained from statistical data and national studies. Aggregation functions are then defined to estimate household's total service unit. The energy consumption (electricity and/or water) is thus calculated stochastically as a function of the service unit and the power rating of the involved appliance. 


\section{APPLICATION OF THE 'SABEC' MODEL ON "WASHING LAUNDRY" ACTIVITY}

In this section, the proposed SABEC model is applied on the domestic activity "washing laundry". First, a description of the activity is given and its different facets are discussed. The modeling logic is then presented and the main variables that influence energy consumption of "washing laundry" activity are exposed. Details on the statistical data being considered, their nature and sources are presented and discussed. Then a demonstration of how the SABEC model can be applied to simulate energy and water consumptions yielded by the considered activity is performed. A number of simulation examples are performed in order to test the model's functionalities. Simulation results are used to interpret the variation in energy consumption among different households. Finally, the proposed model is validated by confronting its results against real measured consumption data.

Due to lack in some statistical data concerning laundry washing habits, a web-based survey was conducted to track the trends of "washing laundry" within French households. 105 respondents from different household types participated in the survey. The results provide us with a comprehensive knowledge base on cloth washing habits in French residential buildings. Some of the statistical data collected from the survey are used in the model.

\subsection{Description of "washing laundry" activity}

Doing laundry at home is one of the major domestic activities since people wash their dirty laundry on a regular basis. The washing machine is a commonly used device and an integral part of most households all over the world. Almost 95\% of French households possess washing machines in their dwelling [33]. On average, a washing machine consumes $169 \mathrm{kWh} /$ year per French household [34], where this value represents about $7 \%$ of French households' total electricity consumption [35]. Different families produce different quantities of dirty laundry, and may use a different number of washing cycles and temperature settings, leading thus to variability in energy consumption.

Doing laundry is the process by which households clean their laundry at home. Laundry materials are composed of both clothes worn by individuals in addition to house linens. We consider the "washing laundry" activity through three different steps: using, sorting and washing, as shown in Figure 2.

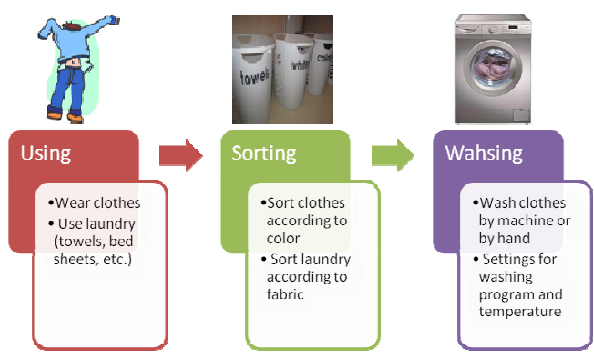

Figure 2 .Representation of "washing laundry" activity

\subsubsection{Using laundry \\ Using clothes}

Each individual wears a quantity of clothes per day. The mean weight of clothes dressed by an French adult per day, denoted by $\overline{Q C}$, is about $1.2 \mathrm{Kg}[36]$.

Using linens

The average quantity of linens owned by French households, denoted by $Q L_{H H}$, is taken from a national study [36] as shown in Table3.
Table 3. AVERAGE QUANTITY OF LINENS PER HOUSEHOLD TYPE

\begin{tabular}{cc}
\hline Household type & Quantity of linens owned (Kg) \\
\hline Single & 6.75 \\
One-parent family & 15.4 \\
Couples without children & 11.45 \\
Couples with children & 16.9 \\
\hline
\end{tabular}

\section{Changing rate}

The changing rate represents the frequency by which individuals put their laundry into dirty-laundry baskets in order to be washed. The conducted study gives some insights about these frequencies. For instance, the different changing rates of clothes for adults and children, together with their probability distributions are presented in Table 4. The changing rate for linens is either once per month $(50 \%)$ or twice $(50 \%)$.

Table 4. CHANGING RATE OF CLOTHES PER INDIVIDUAL

\begin{tabular}{lcc}
\hline Changing rate & Children & Adults \\
\hline Once every day (1) & $69 \%$ & $33 \%$ \\
Once every two days (2) & $23 \%$ & $43 \%$ \\
Once every three days (3) & $\mathbf{8 \%}$ & $24 \%$ \\
\hline
\end{tabular}

\subsubsection{Sorting laundry}

Several studies reveal that people sort their dirty laundry before washing [37,38]. Laundry is in general sorted into dark-colored clothes, light-colored clothes, and linens, where each category is washed at different temperatures. The proportions of light-colored clothes over the total clothes, obtained from our survey, are given as shown in Table5.

\subsubsection{Washing laundry}

Households wash their laundry as a function of its usage and sorting (color) as described previously. The two main parameters of washing laundry are the washing temperature and the filling ratio of machine's drum.

Table 5. DISTRIBUTION OF LIGHT-COLORED CLOTHES PROPORTION (FROM SURVEY)

\begin{tabular}{cc}
\hline Proportion of light-colored clothes & Probability distribution \\
\hline $10 \%$ & $11 \%$ \\
$20 \%$ & $26 \%$ \\
$30 \%$ & $28 \%$ \\
$40 \%$ & $18 \%$ \\
$50 \%$ & $10 \%$ \\
$60 \%$ & $7 \%$ \\
\hline
\end{tabular}

Washing temperature

Elevated washing temperatures induce higher energy consumption than lower ones. A cycle at $90{ }^{\circ} \mathrm{C}$ consumes three times more electricity than a cycle at $30{ }^{\circ} \mathrm{C}$ [37,39]. Different temperatures used for washing light-colored clothes, dark-colored clothes and lines are presented in Table 6 together with their corresponding probability distributions (from survey). It is noticed that high temperatures are mainly used for washing light-colored clothes and linens.

\section{Filling ratio}

The filling ratio is defined as the quantity of laundry that people fill into machine's drum, divided by the machine's nominal capacity. Different households have different filling ratios ranging in general between $50 \%$ and $100 \%$ [37]. The filling ratio has a direct influence on the number of washing cycles per household, and thus on energy 
and water consumption. Different filling ratios results from our survey are presented in Table 7 together with their probability distribution.

Table 6. PROBABILITY DISTRIBUTION OF WASHING TEMPERATURES (FROM SURVEY)

\begin{tabular}{cccc}
\hline & \multicolumn{3}{c}{ Probability distribution } \\
\cline { 2 - 4 } Washing & $\begin{array}{c}\text { light-colored } \\
\text { temperature }\end{array}$ & $\begin{array}{c}\text { dark-colored } \\
\text { clothes }\end{array}$ & linens \\
\hline $30{ }^{\circ} \mathrm{C}$ & $26 \%$ & $48 \%$ & $13 \%$ \\
$40{ }^{\circ} \mathrm{C}$ & $44 \%$ & $44 \%$ & $30 \%$ \\
$60^{\circ} \mathrm{C}$ & $24 \%$ & $8 \%$ & $52 \%$ \\
$90{ }^{\circ} \mathrm{C}$ & $6 \%$ & $0 \%$ & $5 \%$ \\
Total & $100 \%$ & $100 \%$ & $100 \%$ \\
\hline
\end{tabular}

Table 7. DISTRIBUTION OF LIGHT-COLORED CLOTHES PROPORTION (FROM SURVEY)

\begin{tabular}{cc}
\hline Filling ratio of machines drum & Probability distribution \\
\hline $60 \%$ & $6 \%$ \\
$70 \%$ & $4 \%$ \\
$80 \%$ & $24 \%$ \\
$90 \%$ & $43 \%$ \\
$100 \%$ & $23 \%$ \\
Total & $100 \%$ \\
\hline
\end{tabular}

\subsection{Washing machine characteristics}

A washing machine can be characterized by its installation mode (free standing or built in), type (frontal or top), capacity (drum capacity in $\mathrm{Kg}$ ), energy rating (energy class), water intake connection, water and electricity consumption per cycle, and washing programs. The proposed model focuses on modeling activity patterns due to occupants' attributes rather than those due to appliance attributes. For this reason, only two main characteristics of cloth washers, which are machine's charging capacity and energy rating, are considered.

\section{Washing machine's capacity}

The capacity of a washing machine, $C_{W M}$, represents the maximum quantity of laundry that can be charged into machine's drum to be washed through a single cycle. Due to lack in statistical data about capacities of cloth washers within French households, the results of the conducted survey are used (Figure 3).

\section{Washing machine's energy rating}

The energy rating of a washing machine represents its electricity and water consumption levels. The European standard evaluates washing machines' energy rating through classes ranging from $\mathrm{A}+++$ (most efficient) to $\mathrm{G}$ (least efficient).

The energy class corresponds to energy consumption in $\mathrm{kWh}$ per $\mathrm{kg}$ of laundry for the standard cotton cycle at $60{ }^{\circ} \mathrm{C}$, denoted by $P_{W M, 60}{ }^{\circ} \mathrm{C}$. Devices labeled from A to $\mathrm{A}+++$ are considered to be energy-efficient, while others are not. The energy labels and their corresponding energy and water ratings are given in Table 8. Data are taken from studies in [40-42].

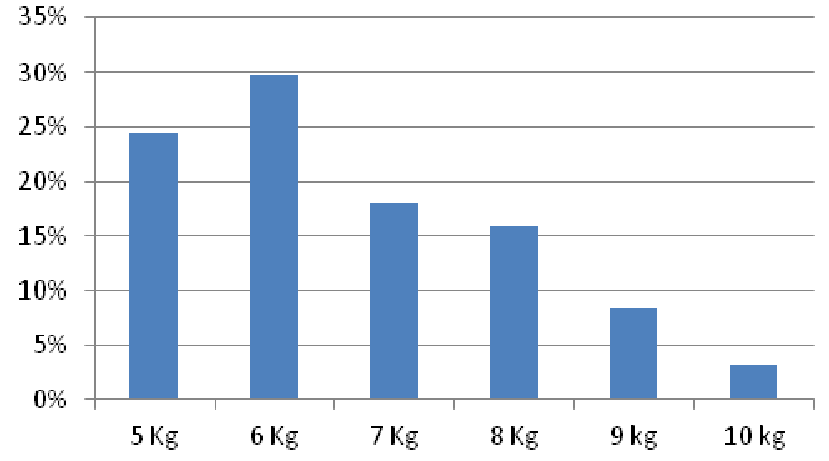

Figure 3 . Probability distribution of the different washing machine capacities (from survey)

Table 8. ENERGY LABELS, THEIR POWER RATINGS AND WATER CONSUMPTION [40-42]

\begin{tabular}{ccc}
\hline Label & $\begin{array}{c}\text { Power rating at } 60{ }^{\circ} \mathrm{C}, \\
P_{W M, 60{ }^{\circ} \mathrm{C}}(\mathrm{KWh} / \mathrm{kg})[\mathrm{min}, \mathrm{max}]\end{array}$ & $\begin{array}{c}\text { Water consumption } \\
(\mathrm{Liter} / \mathrm{kg})\end{array}$ \\
\hline $\mathrm{A}+++$ & {$[0.11,0.13]$} & \\
$\mathrm{A}++$ & {$[0.13,0.15]$} & 7 \\
$\mathrm{~A}+$ & {$[0.15,0.17]$} & \\
$\mathrm{A}$ & {$[0.17,0.19]$} & \\
\hline $\mathrm{B}$ & {$[0.19,0.23]$} & 20 \\
$\mathrm{C}$ & {$[0.23,0.27]$} & \\
$\mathrm{D}$ & {$[0.27,0.31]$} & \\
$\mathrm{E}$ & {$[0.31,0.35]$} & \\
$\mathrm{F}$ & {$[0.35,0.39]$} & \\
$\mathrm{G}$ & {$[0.39,0.43]$} & \\
\hline
\end{tabular}

Having now the energy rating of the machine at $60{ }^{\circ} \mathrm{C}$ form Table 8 , the energy rating at other temperatures can be determined, by using coefficients identified in several measurement campaigns $[37,43]$ as shown in Table 9.

Table 9. DETERMINING POWER RATING FOR EACH WASHING TEMPERATURE

\begin{tabular}{ccl}
\hline & Coefficient & Power consumption $(\mathrm{KWh} / \mathrm{Kg})$ \\
\hline For $60{ }^{\circ} \mathrm{C}$ & 1 & $P_{W M, 60{ }^{\circ} \mathrm{C}}$ (Table 8) \\
For $30{ }^{\circ} \mathrm{C}$ & 0.5 & $P_{W M, 30{ }^{\circ} \mathrm{C}}=P_{W M, 60{ }^{\circ} \mathrm{C}} \times 0.5$ \\
For $40{ }^{\circ} \mathrm{C}$ & 0.66 & $P_{W M, 40{ }^{\circ} \mathrm{C}}=P_{W M, 60{ }^{\circ} \mathrm{C}} \times 0.66$ \\
For $90{ }^{\circ} \mathrm{C}$ & 1.5 & $P_{W M, 90{ }^{\circ} \mathrm{C}}=P_{W M, 60{ }^{\circ} \mathrm{C}} \times 1.5$ \\
\hline
\end{tabular}

Having determined the energy and water consumption of a washing machine per $\mathrm{Kg}$, the energy and water consumption per cycle can now be estimated by multiplying these values with the capacity of the machine. This will be detailed later on.

3.3. Applying SABEC model to estimate electricity and water consumptions of "Washing laundry" activity

In this section, the different steps for calculating energy and water consumptions through SABEC model are exposed. 


\subsubsection{Determining ownership rate of washing machines}

The probability that a given household possess a washing machine $\mathrm{P}(\mathrm{AP})$ is calculated as presented earlier through equation 2 . During a simulation run, a random number is generated and compared to $\mathrm{P}(\mathrm{AP})$ through Monte Carlo technique, so that to determine the ownership state of appliance.

\subsubsection{Determining washing machine's characteristics}

\section{Determining washing machine's energy rating}

The probability that a household possess an energy-efficient appliance $\mathrm{P}(\mathrm{EAP})$ is estimated through equation 3. At each simulation run, a random number is generated and compared to $\mathrm{P}(\mathrm{EAP})$ through Monte Carlo technique, so that to determine whether the owned washing machine is energy-efficient or not. Consequently, another random number is generated to draw uniformly an energy label. Then, the corresponding power rating (at $60^{\circ} \mathrm{C}^{\circ}$ ) of the washing machine is deduced from Table 8 . The power ratings corresponding to other temperatures are then deduced from table 9.

\section{Determining washing machine's capacity}

To determine the capacity of a washing machine $C_{W M}$, the distribution shown earlier in Figure 3 is used. A random number is generated and the capacity is then deduced from this distribution through Monte Carlo technique.

\subsubsection{Determining the service unit of "washing laundry" activity}

The service unit of the activity "Washing laundry" is defined to be the quantity of dirty laundry (clothes and linens) produced by a household per month (in kilograms). Each individual wears a given quantity of clothes per day. This quantity depends mainly on individual's body surface area. The body surface area is a function of humans' height and weight [44], and these are in turn correlated to age and gender.

\section{Service unit per individual}

Given the age and gender of an individual, an estimation of hi/her average height $H_{i}$ can be deduced from national French statistics [45] In addition, the weight of a French individual $W_{i}$ is given through normal probability distributions as a function of individual's age and gender [46].

Given now the height and the weight of an individual, the body surface area $B S A_{i}$ can be calculated as in equation 4 [44].

$B S A_{i}=0.024265 \times W_{i}^{0.5378} \times H_{i}^{0.3964}$

The body surface area of an average French adult, denoted by $\overline{B S A}$, can thus be estimated using average values of weight and height. For males, it is equal to $1.951 \mathrm{~m}^{2}$ while for females it is equal to $1.685 \mathrm{~m}^{2}$. Therefore, given the age and gender of any individual, his/her body surface area can be calculated through equation 4 , and then the quantity of clothes dressed per day $Q C_{i}^{d}$ can be estimated (through rule of three) from the reference values of an adult as shown in equation 5 .

$Q C_{i}^{d}=\left(B S A_{i} \times \overline{Q C}\right) / \overline{B S A}$

Which can be written as

$Q C_{i}^{d}=0.614 \times B S A_{i}$

For males

$Q C_{i}^{d}=0.711 \times B S A_{i}$

For females
To determine thus the quantity of clothes changed by an individual per month $Q C_{i}^{m}$, the formula given in equation 6 is used.

$Q C_{i}^{m}=Q C_{i}^{d} \times\left(30 / C R_{i}\right)$

The changing rate for an individual $C R_{i}$ is generated randomly from the distributions shown earlier in Table 4.

\section{Service unit per household}

The service unit of the activity "washing laundry" for a given household is considered to be additive. This means that the total quantity of clothes laundry per household per month is equal to the sum of all individual quantities as shown in equation 7 .

$Q C_{H H}^{m}=\sum_{i}^{N O} Q C_{i}^{m}$

Where $N O$ is the number of household occupants and $Q C_{i}^{m}$ is the quantity of dirty clothes (to be washed) produced by an individual per month.

We denote by $q$ the percentage of light-colored clothes over the total quantity of clothes per household. During a simulation, a random number is generated and $q$ is estimated from the distribution presented in Table 5. Therefore, the quantity of light-colored clothes, $L C_{h h}^{m}$, and dark-colored clothes, $D C_{h h}^{m}$, to be washed per month by a household are estimated as shown in equations 8 and 9 respectively. In addition, the quantity of linens per household per month is given in equation 10 , where $C R_{l}$ is the changing rate of home linens per month.

$L C_{H H}^{m}=q \times Q C_{H H}^{m}$

$D C_{H H}^{m}=(1-q) \times Q C_{H H}^{m}$

$Q L_{H H}^{m}=Q L_{H H} \times C R_{l}$

\subsubsection{Calculating energy and water consumption}

The first step for calculating energy and water consumption is to determine the washing temperature and the filling ratio of machine's drum (FR). The latter is determined through a random number and using the probability distribution in Table 7. As for washing temperatures, three random numbers are generated randomly to determine respectively washing temperatures for light-colored clothes $\left(\mathrm{T}_{1}\right)$, dark-colored clothes $\left(\mathrm{T}_{2}\right)$, and linens $\left(\mathrm{T}_{3}\right)$ from Table 6 . Therefore, total energy and water consumption of the activity "washing laundry", denoted by $E C_{w m}$ and $W C_{w m}$ respectively, can be calculated as shown in equations 11 and 12 .

$E C_{w m}=\mathrm{EC}_{1}+\mathrm{EC}_{2}+\mathrm{EC}_{3}$

$W C_{w m}=\mathrm{WC}_{1}+\mathrm{WC}_{2}+\mathrm{WC}_{3}$

Where $\mathrm{EC}_{1}$ and $\mathrm{WC}_{1}$ represent the electricity and water consumed for washing light-colored clothes respectively. $\mathrm{EC}_{2}$ and $\mathrm{WC}_{2}$ represent the energy and water consumed for washing dark-colored clothes respectively. $\mathrm{EC}_{3}$ and $\mathrm{WC}_{3}$ represent the energy and water consumed for washing home linens respectively. These are given through equations 13 and 14.

$\mathrm{EC}_{\mathrm{j}}=N C_{j} \times P_{w m, T_{j}}$

$\mathrm{WC}_{\mathrm{j}}=N C_{j} \times \widetilde{W}$

$\mathrm{j}=1$ for light colored clothes, $\mathrm{j}=2$ for dark-colored clothes and $\mathrm{j}=3$ for linens.

Where $\widetilde{W}$ is the average water consumption per cycle, $P_{w m, T_{j}}$ is the power consumption of the washing machine per cycle at a washing 
temperature $T_{j}$ (already determined in section 3.2.2), and $N C_{j}$ represents the number of washing cycles, and calculated as shown in equation 15.

$N C_{j}=\frac{\mathrm{Q}}{F R \times C_{w m}}$

$Q=L C_{H H}^{m}$ for light-colored clothes, $Q=D C_{H H}^{m}$ for dark-colored clothes, and $Q=Q L_{H H}^{m}$ for linens.

\section{TESTING MODEL FUNCTIONALITIES TROUGH SIMULATION EXAMPLES}

For testing the functionality of the model as well as the validity of the results obtained, a number of simulation examples for the three use-cases of the model are performed. These three use-cases are explained briefly hereafter.

\subsection{Use-case 1: simulating energy consumption for specific households}

First of all, the model can be used to quantify the energy consumption of a given activity (here "washing laundry") for a given specific household taken as input. For each simulation, a specific household is defined manually by the user at the entry of the model. For running simulation, five household examples defined by the authors are considered and are described hereafter.

- Household 1: Single person, male, aged 32, active employed, senior profession, with a long-term education level and an income of 2700 Euros/month

- Household 2: Couple without children. Adult 1 is a male aged 37, active employed, senior profession, with long-term educational level and an income of 3000 Euros/month. Adult 2 is a female aged 34 years old, active and employed, middle level professions, with short-term higher education and income of 2300 Euros/month.

- Household 3: Couple with 3 children. Adult 1 is a male aged 45, active employed, clerical and service-staff profession, with a baccalaureate level education and an income of 2000 Euros/month. Adult 2 is a 40 years old female, non-active housewife, with a baccalaureate level education and no salary. The first child is a 9 years old girl, whereas the second and third are boys with 14 and 6 years old respectively. All children go to school

- Household 4: One-parent family with one child. The parent is a 34 years old female, active employed in a middle level profession, with a short-term education level and an income of 1400 Euros/month. The child is a 5 year old boy who goes to school

- Household 5: A couple of retired persons without children. Adult 1 is 66 years old male, inactive retired, Short-term higher education level, and an income of 1300 Euros/month. Adult 2 is a 62 years old female, inactive retired, Baccalaureate education level, and without income.

\subsection{Use-case 2: simulating energy consumption for random households with constraints}

For this second use case, the model can be used to quantify energy consumption of a given activity (here "washing laundry") for a random household taken at the input. The advantage here is that while generating this random household, some constraints can be defined on its attributes. This is an important feature which enables testing variability between households having one or more criteria (attributes) in common.

\subsection{Use-case 3: simulating energy consumption for randomly chosen population of households}

For this third use-case (third functionality of the model), a population of households can be generated randomly by the model. The energy consumption resulting from this third use-case can thus be representative of the total French population. Hence, simulation results can be compared to population-wise real data in order to validate the model.

\section{RESULTS AND DISCUSSIONS}

A number of simulations are performed according to the three use-cases defined in section 4. The results describing energy consumption for the activity "washing laundry" for each use-case are presented in the following.

\subsection{Results for use-case 1}

The model is used to estimate energy and water consumption for each of the five households presented in the previous section. For each household, 10000 simulations are performed where model's probabilistic variables are varied automatically (Appliance ownership, appliance energy rating and characteristics, activity's service unit, etc.). The averages of these results are summarized in Table 10.

Table 10. SIMULATION RESULTS FOR THE FIVE HOUSEHOLDS

\begin{tabular}{cccc}
\hline Household & $\begin{array}{c}\text { Average number } \\
\text { of cycles per } \\
\text { month }\end{array}$ & $\begin{array}{c}\text { Average electricity } \\
\text { consumption } \\
\text { (KWh/month) }\end{array}$ & $\begin{array}{c}\text { Average water } \\
\text { consumption } \\
\text { (Liters/month) }\end{array}$ \\
\hline 1 & 9 & 6.93 & 556 \\
2 & 14 & 10.76 & 849 \\
3 & 26 & 20.57 & 1672 \\
4 & 12 & 11.30 & 968 \\
5 & 15 & 14.60 & 1309 \\
\hline
\end{tabular}

The average results in Table 10 show that household 3 (couple with three children) has the highest consumption values compared to other households. This result is normal since the number of occupants in household 3 ( 5 occupants) is higher than that in others. Moreover, household 1 presents the lowest consumption values. It can be noticed that the number of cycles increases with the increase in the number of occupants, and such do the energy and water consumption. The plot of increasing cumulative frequencies of electricity consumption for the five households is given in Figure 4. This plot shows the difference between electricity consumption values corresponding to each of the five household. For household 3 for example, this consumption can reach $40 \mathrm{KWh} /$ month while it is limited at $14 \mathrm{KWh} /$ month for household 1 .

\subsection{Results for use-case 2}

For this use-case, only two simulation examples are given. In the first example, a constraint is defined on the household type, whereas in the second one, two constraints are defined on the household type and the number of children per household respectively. 


\subsubsection{Use case 2- example 1}

In this example, simulations are performed by defining a constraint on the household type (Single, couples with children, couples without children, and one-parent families). For each household type, 10,000 simulations are performed. For each simulation, the model randomizes the attributes of each individual and then calculates the energy and water consumption yielded by the activity "washing laundry".

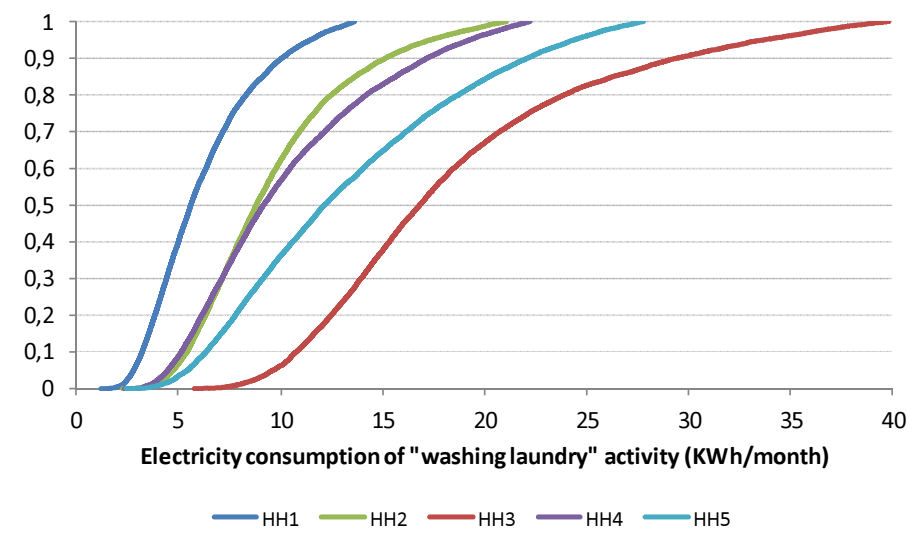

Figure 4 . Increasing cumulative distribution of electricity consumption for the five households

The results are illustrated through the box-plot shown in Figure 5. The lines on each box-plot starting from downside are respectively the minimum value, first quarter, median, third quarter and maximum. The median values of electricity consumption for the respective household types are $15 \mathrm{KWh} /$ month for 'couples with children', $12 \mathrm{KWh} /$ month for 'one-parent families', 10 for 'couples with children' and finally 7 $\mathrm{KWh} /$ month for 'singles'. These findings confirm the direct relation between the size of a household and its corresponding energy and water consumption for the "washing laundry" activity. Large households use more laundry (especially clothes), wash more frequently, and thus consume more electricity and water.

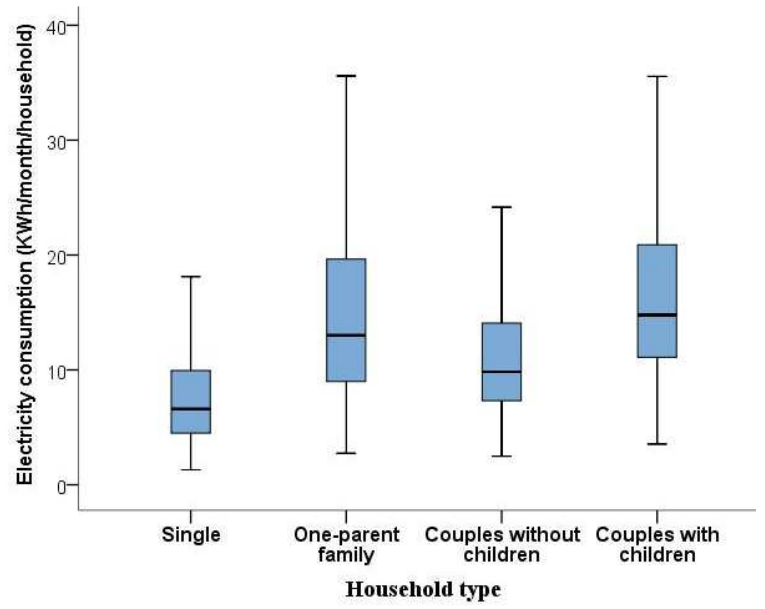

Figure 5 . Electricity consumption simulation results for the four household types (use case 2-example1)

\subsubsection{Use case 2- example 2}

In this example, the model is used to examine energy consumption variation within a homogenous sample of households. Only households of "couples with children" type are considered where a constraint on the number of children is defined. The goal is to analyze consumption variation as a function of the number of children per household. The three cases considered are presented in Table 11.

Table 11. THREE CASES CONSIDERED FOR THE NUMBER OF CHILDREN

\begin{tabular}{lccc}
\hline Case & 1 & 2 & 3 \\
\hline Number of children & {$[1,2]$} & {$[3,4]$} & {$[5,6]$} \\
\hline
\end{tabular}

For each case, ten thousand simulations are performed. The simulation results are illustrated through the box-plot shown in Figure 6.

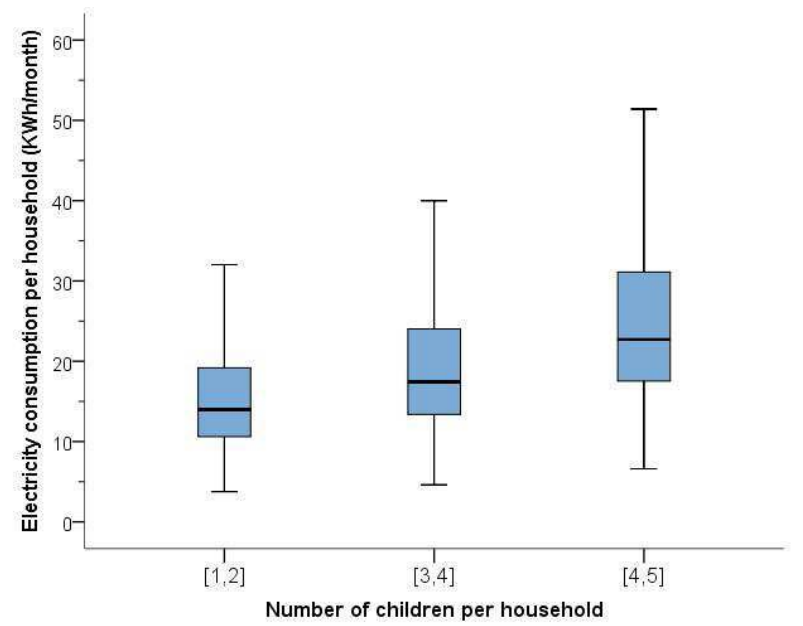

Figure 6 . Electricity consumption simulation results for the four the three cases (use case 2-example 2)

As expected, the electricity consumption of "washing laundry" activity increases linearly with the increase in the number of children per household. Households with 5 to 6 children consume on average 22 $\mathrm{KWh} /$ month for washing laundry, while households with 1 to 2 children do not consume more than $16 \mathrm{KWh} /$ month.

\subsection{Results for use-case 3}

For this case, households are generated randomly according to their probability distributions over the population. In this example, 10,000 random households are generated and their corresponding electricity and water consumptions for the "washing laundry" activity are calculated. For instance, simulation results for water consumption show that the average quantity of water consumed per French household for washing laundry is equal to 871 liters/month. Moreover, the average electricity consumed per household is equal to 12.51 $\mathrm{KWh} /$ month.

\section{Model validation}

In order to validate the model proposed in this paper, simulation results for the energy consumption of the activity 'washing laundry' are compared against real measured data. Water consumption is not confronted here because of the lack of measured data. The real data of energy consumption used by washing machines in French dwellings are taken from a national monitoring study [37], where the histogram 
of electricity consumption of washing machines recorded by the study is shown in Figure 7. In this study, electricity consumption of washing machine was monitored in 87 different households during a period of 44 days. The measurements show that the annual electricity consumption of a washing machine is equal to $169 \mathrm{KWh} /$ year. The extreme consumption values recorded were $850 \mathrm{KWh} /$ year and 34 $\mathrm{KWh} /$ year. The mean electricity consumption is equal to 14.24 $\mathrm{KWh} /$ month while the minimum and maximum values are 2.89 and $70.83 \mathrm{KWh} /$ month respectively.

In order to make comparison with real data, simulation results from model's use-case 3 discussed previously (population-wise) are used. From the 10,000 simulation results, a sample of 87 results (equal to monitored dwellings) is taken. It must be noted here that several samples can be randomly chosen from the 10000 simulation results in possession. For this reason, a number of samplings ( $87 \mathrm{each}$ ) are taken and compared them to each other. The means (average electricity consumption) for all samples are almost similar, yet differences can be witnessed in maximum and minimum values. In The electricity consumption for a sample of 87 households taken arbitrarily from SABEC model's simulation results is also plotted in Figure 7.

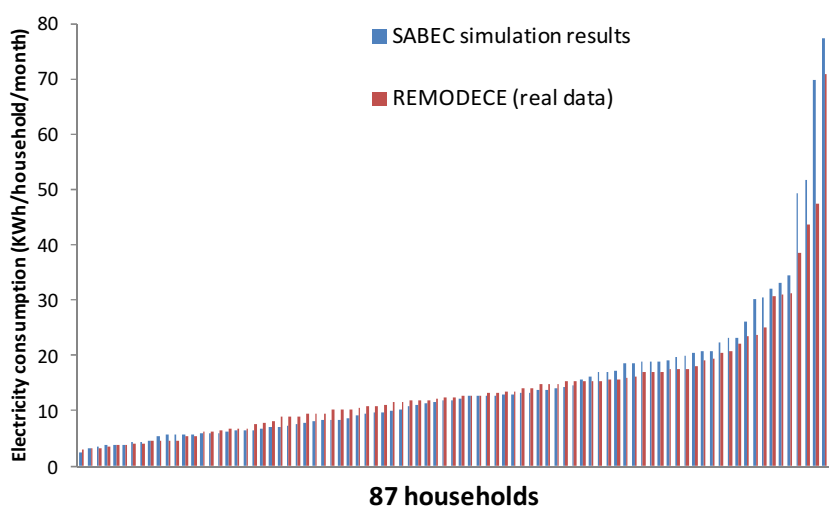

\section{Figure 7 . Electricity consumption of washing machines in 87 French households: SABEC results versus real measured data from [37]}

A first comparison between the energy consumption distribution of simulation results and that of real data is performed through their corresponding descriptive statistics as shown in Table 12.

The mean values $(\mu)$ of both distributions are very close to each other with $\mu=14.98 \mathrm{KWh} /$ month for simulation results and $\mu=14.24$ $\mathrm{KWh} /$ month for real monitored data.

A non-parametric test is also performed to compare the two samples. A Mann-Whitney-Wilcoxon test for independent samples is performed using the SPSS statistical analysis software. The p-value resulting from the test is equal to 0.809 which is favorable thus to retain the null hypothesis, that is the distribution is the same across both samples. This indicates that both samples have similar distributions of electricity consumption values.

Table 12. COMPARISON BETWEEN SIMULATION RESULTS AND REAL DATA

\begin{tabular}{ccc}
\multicolumn{3}{c}{ AND REAL DATA } \\
\hline & \multicolumn{2}{c}{ Electricity consumption $(\mathrm{KWh} /$ household/month) } \\
\cline { 2 - 3 } & Simulation results & Real data from [37] \\
\cline { 2 - 3 } Minimum & 2.33 & 2.89 \\
Maximum & 77.41 & 70.83 \\
Mean $(\mu)$ & 14.98 & 14.24 \\
Standard deviation $\sigma$ & 12.98 & 10.46 \\
\hline
\end{tabular}

The results from the statistical test, coupled with the comparison through descriptive statistics, confirm the similarity of energy consumption distributions for the activity 'washing laundry' between simulation results and real data. These results emphasize the validation of model simulation results, and thus of the SABEC model itself.

\section{Conclusions}

In this paper, a modeling approach which gives a probabilistic mapping between household profiles and their corresponding domestic energy consumption is proposed. A bottom-up model based on individual domestic activities and appliances is adopted. The stochastic activity-based model (SABEC) is exposed together with its different variables. An application example of the SABEC model is then demonstrated on the activity "washing laundry". A number of simulations are performed so that to demonstrate the different functionalities of the model. Three simulation types of energy consumption are demonstrated: for specific households, for random households with constraints, and finally for population-wise random households. Simulation results for the "washing laundry" activity" are then presented and discussed. These results show a good similarity with real national monitored electricity consumption data. The advantage of the model in assessing consumption variability between different households with different attributes is then highlighted. Finally, the model is validated by confronting its simulation results to real measured data. It must be noted here that unfortunately in literature, we didn't find an approximate modeling approach which is applied to similar population (French) so that to compare its simulation results with those yielded by the SABEC model presented here.

For this instance, the SABEC model was only applied on two domestic activities, namely watching TV and washing laundry. A framework to generalize the model on other domestic activities is already sketched. Moreover, efforts for simplifying of the model are being conducted. For this sake, sensitivity analysis is used to identify the most influencing input variables of the model. This is already done for the "washing laundry" activity, where it is revealed that the major occupant-related factors influencing energy consumption of this activity are households' number of adults, number of children, and total income. The details of this study are not included in this paper due to space limitation.

The major features of the proposed model can be summarized by its capability, first to produce energy consumption estimates with a high granularity (per household and per activity), and secondly to assess energy consumption variability between different households with different attributes. Future works, beside model's generalization and simplification, include also the development of a simulation tool which can be later integrated into the design process of buildings to help experts assessing detailed consumption trends of buildings.

\section{REFERENCES}

[1] Saidur, R., Masjuki, H. H., and Jamaluddin, M. Y., 2007, "An application of energy and exergy analysis in residential sector of Malaysia," Energy Policy, 35(2), pp. 1050-1063.

[2] Masoso, O. T., and Grobler, L. J., 2010, "The dark side of occupants" behaviour on building energy use," Energy and Buildings, 42(2), pp. $173-177$.

[3] ADEME, 2013, http://www2.ademe.fr [Online]. Available: http://www2 ademe.fr/servlet/KBaseShow?sort=$1 \&$ cid $=96 \& \mathrm{~m}=3 \&$ catid $=17778$

[4] Yu, Z., Fung, B. C. M., Haghighat, F., Yoshino, H., and Morofsky, E., 2011, "A systematic procedure to study the influence of occupant 
behavior on building energy consumption," Energy and Buildings, 43(6), pp. 1409-1417.

[5] Fabi, V., Andersen, R., Corgnati, S., and Olesen, B., 2012, "Occupants' window opening behaviour: A literature review of factors influencing occupant behaviour and models," Building and Environment, 58, pp 188-198.

[6] Fischer, M., and Kunz, J., 2004, "The scope and role of information technology in construction," Proceedings-Japan Society of Civil Engineers, pp. 1-32.

[7] Malavazos, C., Tzovaras, D., Kehagias,, D., and Ioannidis, D., 2011, "ENERGY AND BEHAVIOURAL MODELLING AND SIMULATION FOR EE-BUILDINGS DESIGN."

[8] Kashif, A., Ploix, S., Dugdale, J., and Binh Le, X. H., 2012, "Simulating the dynamics of occupant behaviour for power management in residential buildings," Energy and Buildings.

[9] Swan, and Ugursal, 2009, "Modeling of end-use energy consumption in the residential sector: A review of modeling techniques," Renewable and Sustainable Energy Reviews, 13(8), pp. 1819-1835.

[10] Page, J., Robinson, D., Morel, N., and Scartezzini, J. L., 2008, "A generalised stochastic model for the simulation of occupant presence," Energy and Buildings, 40(2), pp. 83-98.

[11] Robinson, D., 2006, "Some trends and research needs in energy and comfort prediction," Windsor Conference

[12] Lutzenhiser, L., and Bender, S., 2008, "The 'Average American' Unmasked: Social Structure and Differences in Household Energy Use and Carbon Emissions."

[13] Guerin, D. A., Yust, B. L., and Coopet, J. G., 2000, "Occupant predictors of household energy behavior and consumption change as found in energy studies since 1975," Family and Consumer Sciences Research Journal, pp. 48-80.

[14] McLoughlin, F., Duffy, A., and Conlon, M., 2012, "Characterising domestic electricity consumption patterns by dwelling and occupant socio-economic variables: An Irish case study," Energy and Buildings, 48, pp. 240-248

[15] Swan, L. G., and Ugursal, V. I., 2009, "Modeling of end-use energy consumption in the residential sector: A review of modeling techniques," Renewable and Sustainable Energy Reviews, 13(8), pp 1819-1835.

[16] Pachauri, S., 2004, "An analysis of cross-sectional variations in total household energy requirements in India using micro survey data," Energy Policy, 32(15), pp. 1723-1735.

[17] Seryak, J., and Kissock, K., 2003, "Occupancy and behavioral affects on residential energy use," Proceedings of the Solar Conference, pp. $717-722$.

[18] Chiou, Y.-S., 2009, "Deriving US household energy consumption profiles from american time use survey data a bootstrap approach," 11 th International Building Performance Simulation Association Conference and Exhibition, Glasgow, Scotland, pp. 151-158.

[19] Tanimoto, J., Hagishima, A., and Sagara, H., 2008, “A methodology for peak energy requirement considering actual variation of occupants' behavior schedules," Building and Environment, 43(4), pp. 610-619

[20] Tanimoto, J., Hagishima, A., and Sagara, H., 2008, "Validation of probabilistic methodology for generating actual inhabitants' behavior schedules for accurate prediction of maximum energy requirements," Energy and Buildings, 40(3), pp. 316-322.

[21] Richardson, I., Thomson, M., and Infield, D., 2008, "A high-resolution domestic building occupancy model for energy demand simulations," Energy and Buildings, 40(8), pp. 1560-1566.

[22] Richardson, I., Thomson, M., Infield, D., and Clifford, C., 2010, "Domestic electricity use: A high-resolution energy demand model," Energy and Buildings, 42(10), pp. 1878-1887.

[23] Widén, J., and Wäckelgård, E., 2010, "A high-resolution stochastic model of domestic activity patterns and electricity demand," Applied Energy, 87(6), pp. 1880-1892.

[24] Muratori, M., 2012, "A Highly Resolved Modeling Technique to Simulate Residential Power demand," Applied Energy, Preprint submitted.

[25] Subbiah, R., 2013, “An Activity-Based Energy Demand Modeling Framework for Buildings: A Bottom-Up Approach," Virginia Polytechnic Institute and State University.
[26] Quijano, J., Herpson, C., and Sabouret, N., 2010, "Prédiction de l'activité humaine afin de réduire la consommation électrique de l'habitat," pp. 43-52.

[27] Druckman, A., and Jackson, T., 2008, "Household energy consumption in the UK: A highly geographically and socio-economically disaggregated model," Energy Policy, 36(8), pp. 3177-3192.

[28] Barr, S., Gilg, A. W., and Ford, N., 2005, "The household energy gap examining the divide between habitual- and purchase-related conservation behaviours," Energy Policy, 33(11), pp. 1425-1444.

[29] INSEE, 2012, "Insee - Conditions de vie-Société - Enquête Emploi du temps 2009-2010" [Online]. Available: http://www. insee.fr/fr/themes/document.asp?ref id=edt2010.

[Accessed: 03-May-2013]

[30] Maresca, B., Picard, R., and Dujin, A., 2009, La consommation d'énergie dans l'habitat entre recherche de confort et impératif écologique, Centre de recherche pour l'étude et l'observation des conditions de vie.

[31] INSEE, 2013, "National Institute of Statistics and Economic Studies France" [Online]. Available: http://www.insee.fr/en/default.asp. [Accessed: 07-Mar-2013].

[32] Journel, A. G., 2002, "Combining knowledge from diverse sources: An alternative to traditional data independence hypotheses," Mathematical geology, 34(5), pp. 573-596

[33] INSEE, 2010, "Insee - Conditions de vie-Société - Équipement des ménages en biens durables selon le type de ménage" [Online]. Available:

http://www.insee.fr/fr/themes/tableau.asp?reg_id=0\&ref_id=NATnon05 155. [Accessed: 21-Feb-2013].

[34] SIDLER, O., 2009, Connaissance et maîtrise des usages specifiques de l'electricite dans le secteur residentiel, ENERTECH.

[35] ADEME, 2012, "Maîtriser et limiter leur consommation à la maison: Gérer ses équipements électriques."

[36] Tout Pratique, 2013, "Poids des vêtements et du linge - Tout Pratique" [Online]. Available: http://www.toutpratique.com/6-Vetementsaccessoires/87-Lavage-en-machine-l-eau-debordements-economieslessives-et-assouplissants/5270-Poids-des-vetements-et-du-linge.php. [Accessed: 26-Nov-2013].

[37] Enertech, EDF, and ADEME, 2008, Campagne de mesures des appareils de production de froid et des appareils de lavage dans 100 logements, Project AEE2008.

[38] Roberts, P., 2012, "Yara valley future Water: 2011 appliance stock and usage patterns survey."

[39] Bosch, 2013, "Lave-linge: Quelle classe énergétique choisir?" [Online]. Available: http://www.bosch-home.be/fr/services/bienchoisir/lave-linge/3-consommation.html. [Accessed: 25-Nov-2013]

[40] ECDGE, 2013, "Energy: Energy Labelling of Products - European Commission," European Commission's Directorate-General for Energy [Online].

http://ec.europa.eu/energy/efficiency/labelling/labelling_en.htm. [Accessed: 10-Nov-2013]

[41] GIFAM, 2012, Le lave-linge, Interprofessional group of manufacturers of domestic appliances (GIFAM).

[42] Picard, G., 2008, Lave linge, 60 million de consommateurs

[43] ADEME, 2010, Le lavage domestique, French Environment and Energy Management Agency (ADEME), http://www2 ademe.fr.

[44] Haycock, G. B., Schwartz, G. J., and Wisotsky, D. H., 1978 , "Geometric method for measuring body surface area: A height-weight formula validated in infants, children, and adults," The Journal of Pediatrics, pp. 62-66

[45] GFA, 2013, "Le Groupe Français d'Auxologie" [Online]. Available: http://www.auxologie.com/croissance/. [Accessed: 24-Dec-2013]

[46] Tanguy, J., Zeghnoun, A., and Dor, F., 2007, "Description du poids corporel en fonction du sexe et de l'âge dans la population française," Environnement, Risques \& Santé, 6(3), pp. 179-187. 